\title{
CONDUCTANCE OF A QUANTUM WIRE WITH FINITE LENGTH
}

\author{
M. Suhrke, S. Wilke and R. Keiper \\ Department of Physics, Humboldt University Berlin, Invalidenstr. 110, Berlin 1040, \\ Germany
}

(Received August 8, 1990)

The conductance of a ballistic quantum wire between two rescrvoirs exhibits steps of height $2 e^{2} / h$, if the occupation of transverse subbands is changed. We investigate conditions for observation of these steps starting from Kubo-Greenwood formula. We show how the conductance steps are influenced by the properties of the external regions as well as by the nature of the connection between these regions and the wire. Furthermore we incorporate residual scattering in long wires.

PACS numbers: $72.10 . \mathrm{Bg}, 72.20 . \mathrm{Fr}, 73.40 . \mathrm{Lq}$

The experimental observation of the quantization of the conductance of a narrow constriction in a 2DEG [1] has been the starting point for a variety of experiments as well as theoretical calculations for the conduction of quasi 1D structures in the ballistic regime.

In this paper we investigate the conditions for the observation of structures in the conductance of a wide-narrow-wide (WNW) structure related to $1 \mathrm{D}$ subbands. We start from Kubo-Greenwood formula in real space representation for a two-point measurement [2]. That means, we assume that the voltage drops in the narrow region of length $L$ exclusively and its asymptotic values are measured in the wide regions.

In a small structure the resistance becomes a non-local quantity due to the phase coherence of the wave functions. Consequently, the conductance is influenced by the properties of the structure within a phase coherence length which is larger than $L$ usually. However, as can be shown [2], the total voltage drops only and not the details of the electric field profile enter the total current. Furthermore the properties of the external regions can be easily described via boundary conditions at the wide-narrow (WN) interfaces [3]. Thus, we may consider different residual scattering in the wide and narrow regions which is assumed to be local in space and is calculated within self-consistent Born approximation (self-energy $\Sigma_{\mathbf{B}}(E)$ ). 

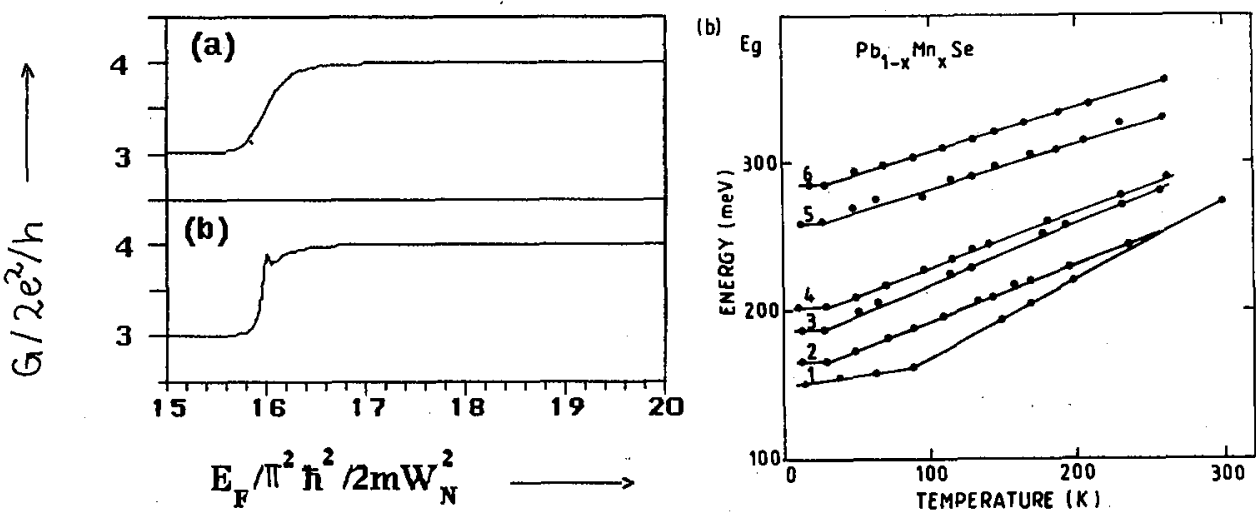

Fig. 1. Conductance steps at the fourth subband bottom with uniform residual scattering (a) and for residual scattering in the narrow region only (b) for a wire of intermediate length.

Following [2] the two-point conductance of the structure is given by

$$
G=I / V=\frac{e^{2} h}{m^{2}} \int_{S} \mathrm{~d}^{2} \bar{r}_{\perp} \mathrm{d}^{2} \bar{r}_{\perp}^{\prime} A\left(\bar{r}, \bar{r}^{\prime} ; E_{\mathrm{F}}\right) \stackrel{\leftrightarrow}{\nabla} \vec{r} \stackrel{\leftrightarrow}{\nabla}_{\bar{r}^{\prime}} A\left(\bar{r}^{\prime}, \bar{r} ; E_{\mathrm{F}}\right)
$$

neglecting thermal broadening. Here, $\vec{\nabla}_{\bar{r}}=\frac{1}{2}\left(\vec{\nabla}_{\bar{r}}-\overleftarrow{\nabla}_{\bar{r}}\right)$ and $A$ is the spectral function in real space. First we consider the adiabatic case, i.e. perfect matching between the subbands in the wide and narrow regions neglecting scattering at the WN interface. Within a diagonal subband expansion the result for the conductance is

$$
G=\frac{2 e^{2}}{h} \sum_{\alpha} \frac{\left(\operatorname{Re} \kappa_{\alpha}\right)^{2}}{\left(\operatorname{Re} \kappa_{\alpha}\right)^{2}+\left(\operatorname{Im} \kappa_{\alpha}\right)^{2}}=e^{2} \sum_{\alpha} v_{\alpha}^{\mathrm{F}} n_{\alpha}\left(E_{\mathrm{F}}\right)
$$

with $\kappa_{\alpha}=\left[\frac{2 m}{\hbar^{2}}\left(E_{\mathrm{F}}-E_{\alpha}-\hbar \sum_{\mathrm{B}}^{R}\left(E_{\mathrm{F}}\right)\right)\right]^{1 / 2}, \operatorname{Re} \kappa_{\alpha}, \operatorname{Im} \kappa_{\alpha}>0$ where $\alpha$ labels the 1D subbands in the narrow region. For vanishing residual scattering this leads to perfect quantization of the two-point conductance

$$
G=\frac{2 e^{2}}{h} \sum_{\alpha} \Theta\left(E_{\mathrm{F}}-E_{\alpha}\right)
$$

due to the cancellation of the energy dependence of the subband DOS $n_{\alpha}$ and the subband velocity $v_{\alpha}$ in the ideal ballistic regime. Uniform residual scattering broadens the steps but does not lead to any additional structures in the conductance (Fig. 1a). In general, non-adiabatic case we use the technique of surface Green functions [3] to match the unperturbed Green functions of the ideal wide and narrow regions. Scattering of waves at the WN interfaces is described by a matrix $R$ given by the unperturbed Green functions and their first derivatives at 
the interfaces. The external wide parts of the structure thus act as a new boundary condition. Within a diagonal subband expansion the coefficients of the matrix $R$ have the structure

$$
r_{\alpha \alpha} \propto\left[1-p_{\alpha}^{2} \exp \left(2 \mathrm{i} \kappa_{\alpha}^{N} L\right)\right]^{-1}, p_{\alpha}=\frac{\kappa_{\alpha}^{N}-\kappa_{\alpha}^{W}}{\kappa_{\alpha}^{N}+\kappa_{\alpha}^{W}}
$$

where $\left(\kappa_{\alpha}^{W}\right)^{-1}=\sum_{j}\left|c_{j}^{\alpha}\right|^{2}\left(\kappa_{j}^{W}\right)^{-1}$ arises from the expansion of the subbands $\alpha$ in the narrow region with respect to those of the wide regions labelled by $j$. Thus, sharp structures in the conductance are expected to be due to resonances in $r_{\alpha \alpha}$ for small denominator. As an effect of the mismatch of the wave functions the effective wave vectors $\kappa_{\alpha}^{W}$ are complex quantities even for vanishing residual scattering.

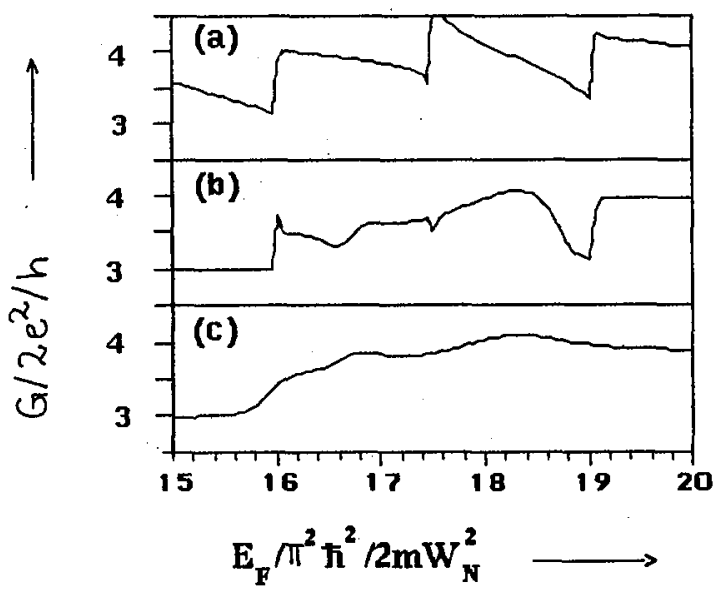

Fig. 2. Conductance step at the fourth subband bottom for $W_{W} / W_{N}=5.5$ (a) short constriction, no residual scattering; (b) wire of intermediate length, no residual scattering; (c) wire of intermediate length, uniform residual scatering.

Within this approach we analyse first the effect of residual scattering in the narrow region only. This nonuniformity results in the reflection of waves at the WN interfaces and leads to the sharp antiresonance in the conductance at subband bottoms indicated in Fig. 1b. This is the consequence of the strong scattering at each subband bottom $\alpha$ which gives $p_{\alpha}=1$.

Secondly, we consider non-adiabaticity due to geometrical inhomogeneity leading to sharp structures superimposed onto the conductance steps. These oscillations reflect the subband structure of the wide regions and are especially pronounced for very short constrictions due to the tunnelling of evanescent states (Fig. 2a, steps related to the 21st and 22nd subband of the wide regions are seen additionally to that of the fourth subband of the narrow region). In a wire of intermediate length there is an additional structure at the bottom of the fourth subband connected with the formation of longitudinal standing waves inside the 
wire (Fig. 2b). The reduced tunnelling gives rise to a decrease of the conductance steps arising from the wide subbands but the antiresonances from $r_{\alpha \alpha}$ remain. Finally, finite residual scattering immediately destroys the resonances and fattens the steps. A comparison of Fig. 1 and $2 \mathrm{c}$ shows that intermadiate residual scattering reduces the sensitivity to boundary conditions but preserves an approximate quantization as indicated by the experiments, too.

\section{References}

[1] B.J. van Wees, H. van Houten, C.W.J. Beenakker, J.G. Williamson, L.P. Kouwenhoven, D. van der Marel, C.T. Foxon, Phys. Rev. Lett. 60, 848 (1988);

D.A. Wharam, T.J. Thornton, R. Newbury, M. Pepper, II. Ahmed, J.E.F. Frost, D.G. IIasko, D.C. Peacock, D.A. Ritche, G.A.C. Jones, J. Phys. C 21, L209 (1988).

[2] D.S. Fisher, P.A. Lee, Phys. Rev. B 23, 6851 (1981);

A.D. Stone, A. Szafer, IBM J. Res. Dev. 32, 384 (1988);

II.U. Baranger, A.D. Stone, Phys. Rev. B 40, 8169 (1989).

[3] F. Garcia-Moliner, V.R. Velasco, Progr. Surf. Sci. 21, 93 (1986). 